\title{
Editorial
}

\author{
M. N. Hoda ${ }^{1}$
}

Published online: 23 February 2022

(C) The Author(s), under exclusive licence to Bharati Vidyapeeth's Institute of Computer Applications and Management 2022

Warm greetings to all our readers!!! We hope the upcoming year brings relief and progress to humanity. BJIT remains committed to delivering on its challenge of consistently showcasing and disseminating novel researches pertaining to computing applications and capable of altering the quality of human life. It is a matter of great privilege for me to unveil before you the fortieth issue i.e. Volume 14 Number 02 of the "International Journal of Information Technology" [An official Journal of Bharati Vidyapeeth's Institute of Computer Applications and Management (BVICAM), New Delhi] with acronym BJIT. From this volume onwards we have increased our frequency to seven issues an year. The issue is live on the Springer content platform SpringerLink and available to the prospective readers through Springer CS package globally.

Throughout the world, nations have started recognizing that Information Technology (IT) is now acting as a catalyst in speeding up the economic activities in efficient governance, citizens' empowerment, sustainable development and in improving the quality of human life. Recent advancements in IT have touched almost every conceivable area of human life. Its degree of pervasiveness, in day to day life, is rapidly increasing, every new day. On the backdrop of this, BJIT has accepted the challenge to consistently showcase, disseminate and institutionalize the rapidly changing huge knowledgebase globally, with authenticity and accuracy, having special focus on the new researches pertaining to IT applications for improving the quality of day to day life.

\footnotetext{
M. N. Hoda

bjit@bvicam.ac.in

1 BJIT, New Delhi, India
}

Volume 14 Number 02 presents a compilation of fifty papers, chosen out of over 500 manuscripts, that span a broad variety of topics from various emerging areas of Information Technology and Computer Science, especially addressing current research problems related to deadlock free systems, data security, wireless sensor networks, online searching, multimodal biometric, cloud services and plant disease identification; to name a few.

Rapid detection of sub-networks in a network is essential to gauge the functionality of a network. The first manuscript in this issue "Assessing the impact of the density and sparsity of the network on community detection using a Gaussian mixture random partition graph generator", Ashani Wickramasinghe et al. evaluates proof-of-concept of a novel random graph generator through a mixture of Gaussian distributions. The second manuscript "Traffic flow prediction using support vector regression", Nidhi et al. propositions a hybrid traffic flow prediction technique. The next manuscript "A novel resource management technique for deadlock-free systems", Madhavi Devi Botlagunta et al. outlines a novel resource reservation technique to estimate the optimal number of resources required for a deadlock free resource reservation policy. The manuscript "Bifold-crypto-chaotic steganography for visual data security", Bharti Ahuja et al. implements a hybrid algorithm to protect data from multiple types of hackers. The manuscript "Modelling and predicting software vulnerabilities using a sigmoid function", Javaid Iqbal et al. proposes an improved timebased model for vulnerability discovery process. The next manuscript "Euler totient function and fermat Euler theorem based an optimized key management scheme for securing mobile agents migration", Pradeep Kumar et al. intends the issue of agent security and its authentication during the hoping of agents in different 
platforms through a mathematical key management technique. The manuscript "On-demand charging planning for WRSNs based on weighted heuristic method", Naween Kumar et al. contends a heuristic charging scheme aimed at maximising sensor lifetimes. The next manuscript "Online searching trend on Covid-19 using Google trend: infodemiological study in Malaysia", Tengku Adil Tengku Izhar et al. advises future action regarding Covid outbreak by monitoring public searching activities on Covid-19. The manuscript "A novel approximate PageRank computation: QEGauss-Seidel PageRank", Atul Kumar Srivastava et al. addresses a novel a low-cost, functional extrapolation method for PageRank computation, in conjunction with the GaussSeidel method. To cimprove the spectral efficiency of optical systems. The manuscript "Comparative analysis of capacity oriented Integrated AMI modulation technique for the deployment of radio over fiber system", Namita Kathpal et al. captures the details of a novel, advanced modulation technique based upon external modulation (electro-absorption modulator). The manuscript "Secure and efficient multi-tenant database management system for cloud computing environment", G.B. Pallavi et al. suggests a novel, secure and efficient multi-tenant database management system for cloud computing environment. The manuscript "A literature review: various learning techniques and its applications for eye disease identification using retinal images", Vipul Rajyaguru et al. delineates the detection of glaucoma using various learning models based on retinal images. Unimodal biometrics is a popular technology for analyzing the physical traits of a human being. The manuscript "A multimodal biometric system using match score and decision level fusion", Prachi Punyani et al. propagates a modified method for biometric identification. The manuscript "Lower-part approximate multi-bit adders for low-power DSP", Chinna Gowdar et al. captures novel lower-part approximate multi-bit adders. The manuscript "A novel web ranking algorithm based on pages multi-attribute", Mohammad Rashad Baker et al. offers an intelligent ranking algorithm based on page multi-attribute. The manuscript "Comparative performance analysis of GFDM and UFMC under different window constraints for next generation cognitive radio communication", Manisha Gupta et al. details performance of two important techniques for next generation cognitive radio communication. The manuscript "IoT infrastructure for the accident avoidance: an approach of smart transportation", Hitesh Mohapatra et al. presents a cross-point collision avoidance model for better predictability about neighboring vehicles and road-crossing points. Apple is an important fruit in India. The manuscript "Wireless sensor network in precision farming for forecasting and monitoring of apple disease: a survey", Firasath Nabi et al. details a analysis of various systems in precision farming for monitoring apple disease. The manuscript "SCGKM: a secure and cost-effective group key management scheme for multicast communication in large dynamic groups", Vinod Kumar et al. develops an efficient multicast communication scheme. The manuscript "Quality of service aware routing protocols in wireless multimedia sensor networks: survey", Roanld Chiwariro et al. evaluates characteristics and requirements of wireless multimedia sensor networks and approaches to mitigating existing challenges. The manuscript, "An empirical analysis of cloud based robotics: challenges and applications", Mimansha Saini et al. analyzes the different applications of robotics deployed in recent times on to the cloud platform. E \& M-governance has seen a massive rise in developed countries. The manuscript, "Spatial Role Labelling in Arabic using Probabilistic Classifiers", Salha Alzahrani et al. investigates the concept of spatial role labeling on Arabic text through probabilistic classifiers. The manuscript, "Energy efficient intra-cluster data aggregation technique for wireless sensor network", Anupkumar M. Bongale et al. details an intra-cluster data aggregation technique for wireless sensor networks. The manuscript, "HONEYDOS: a hybrid approach using data mining and honeypot to counter denial of service attack and malicious packets", Pratima Sharma et al. evaluates a novel hybrid approach for preventing Denial of Service Attack. The next manuscript, "Virtualization vulnerabilities, security issues, and solutions: a critical study and comparison", Darshan Tank et al. empirically analyses virtualization specific threats, vulnerabilities and issues. The manuscript, "An efficient average execution time-round-robin (AET-RR) scheduling algorithm”, Sudhansu Bala Das et al. collates a novel average execution time-round-robin algorithm for both online and offline scenario with varying time quantum. Visible light is intrinsically suited to vehicle to vehicle communication. The manuscript," Investigating the performance of a vehicular communication system based on visible light communication (VLC)", Sumita Mishra et al. investigates a novel a vehicle to vehicle communication system based on visible light communication. The manuscript, "Improved grey relational analysis-based TOPSIS method for cooperation enforcing scheme to guarantee quality of service in MANETs ", Jagatheswari S. et al. introduces a new, improved grey relational analysis-based TOPSIS method as a cooperation enforcing strategy to assess the degree of trust rendered by each mobile node in the network. The manuscript, "Revisiting enterprise resource planning (ERP) risk factors over the past two decades: defining parameters and providing comprehensive 
classification", Babak Zendehdel Nobari et al. offers a comprehensive categorization of factors and effective parameters of enterprise resource planning systems. The manuscript, "T-SEA: trust based secure and energy aware routing protocol for mobile ad hoc networks", Deepika Kukreja et al. details a routing protocol for detection and isolation of black/gray hole nodes in mobile ad hoc networks. The manuscript, "Classification of leaves of medicinal plants using laws' texture features", Diksha Puri et al. characterizes the different leaves of the medicinal plants using computer aided classification system. The next manuscript, "Efficient resource allocation scheme for on-the-fly computing based mobile grids", Amit Sadanand Savyanavar et al. builds an efficient resource allocation model which provides resource allocation with failure handling. The manuscript, "Group security using ECC", Purna Sethi et al. outlays a group security algorithm using the ECC cryptography algorithm. The manuscript, "Discrimination of multi-class EEG signal in phase space of variability for epileptic seizure detection using error correcting output code (ECOC)", Salim Rukhsar identifies a novel mechanism for detection of epileptic seizures using ensemble learning. Load balancing is a significant challenge of the cloud network. The manuscript, "Efficient load balancing techniques for multi-datacenter cloud milieu", Sumanta Chandra Mishra et al. proposes two multi-datacenter two-phase load adjustment techniques for load balancing and apposite task scheduling. The manuscript, "Design of fault tolerant bifunctional parity generator and scalable code converters based on QCA technology", Nuriddin Safoev et al. empirically details a novel designs for digital code converter and parity generator circuits in quantum-dot cellular automata (QCA) technology, which is transistor less computation approach and encodes binary information through the configuration of charges among quantum dots. The manuscript, "Design of cognitive radio system and comparison of modified whale optimization algorithm with whale optimization algorithm", Sumit Bansal et al. emulates and proposes an improved population based meta-heuristics optimization technique to fulfill the objectives of cognitive radio system. The next manuscript, "A case study on AGV's alternatives selection problem", Divya Agarwal et al. simulates the performance of automated guided vehicle techniques. Effective fingerprint identification is an important research concern. The manuscript, "Automatic latent fingerprint identification system using scale and rotation invariant minutiae features", Uttam U. Deshpande simulates the new clustered minutiae-based scale and rotation invariant fingerprint matching method. The next manuscript, "Open source software: analysis of available reliability models keeping security in the forefront", Shiva Tyagi et al. evaluates some different methods to simplify the task of the users in choosing the right model with right approach for the specific required Open Source Software. Technical debt is the debt in software development. The manuscript, "Understanding factors affecting technical debt", Jaspreet Bedi et al. elaborates a novel approach to identify the most contributing factors effecting technical debt. The manuscript. "Empirical analysis of synthetic and real networks", Pranav Nerurkar et al. analyzes the performance of network theoretic tools on common data sets. Cloud computing is being adopted by many governments to ensure efficiency. The manuscript, "A validation of security determinants model for cloud adoption in Saudi organisations' context", Madini O. Alasaffi et al. o investigates the impact of various independent security related factors on the adopted security taxonomy. The next manuscript, "IoT-Blockchain driven traceability techniques for improved safety measures in food supply chain", S. Balamurugan outlays a novel mechanism solution to solve safety, quality and traceability problems in food products. The manuscript, "Efficiency measures for ranked pages by Markov Chain Principle", Swati Jain et al. details an ideal technique for link analysis. The manuscript, "Optimized data transmission scheme based on proper channel coordination used in vehicular ad hoc networks", Aradhana Behura proposes a model for reducing the collision probability when the vehicle node increases in the road. Agile software development has found wide adoption across organizations. The manuscript, "Identification and integration of security activities for secure agile development", Amit Sharma et.al. extends a new framework for secure agile development. The manuscript, "Analysing and securing the sustainability of e-Government projects from technical aspect using employees perspective approach", Jyoti Yadav et al. details and analyzes sustainability of various e-government initiatives. The manuscript, "Understanding structure and behavior of systems: a network perspective", Pranav Nerurkar prototypes behavior of systems in a network scenario. The last manuscript, "Image retrieval system based on multi feature extraction and its performance assessment", R. Tamilkodi et al. outlines three different methods to retrieve images based on the unique features of an image.

I am sure the contributions in this issue, which is an amalgamation of novel trends and technologies to improve our life and sustainability in the present environment, will not only enrich our reader's knowledgebase but will also motivate many of the potential researchers to take up these challenging application areas and contribute effectively for the overall prosperity of the mankind.

As a matter of policy, all the manuscripts received and considered for the Journal, are double blind peer reviewed 
by at-least two independent referees. Our panel of expert referees posses a sound academic background and have a rich publication record in various prestigious journals representing Universities, Research Laboratories and other Institutions of repute, globally. Finalizing the constitution of the panel of referees, for double blind peer review(s) of the considered manuscripts, was a painstaking process, but it helped us to ensure that only the best, interesting and novel of the considered manuscripts are showcased and that too after undergoing multiple cycles of review, as required.

I wish to express my sincere gratitude to the entire editorial board, members of the resident editorial team and our panel of experts in steering the considered manuscripts through multiple cycles of review and bringing out the best from the contributing authors. I thank my esteemed authors for having shown confidence in BJIT and considering it a platform to showcase and share their original research work. I would also wish to thank the authors whose papers could not have been published in this issue of the Journal, probably because of the minor shortcomings. However, I would like to encourage them to actively contribute for the forthcoming issues.

I will fail in my duty, if I do not thank the members of the team from the Springer, particularly Ms. Suvira Srivastav, Mr. Madan Ellappan, Ms. Jeyapradha Saravanan, Ms. Teena Bedi and Ms. Nidhi Chandok for their constant support in realizing the issue and presenting it before you.

The undertaken Quality Assurance Process involved a series of well defined activities that, I trust, went a long way in ensuring the quality of the publication. Still, there is always a scope for improvement, and so, I request the contributors and readers to kindly mail me their criticism, suggestions and feedback at bjit@bvicam.ac.in and help in further enhancing the quality of forthcoming issues.

\section{N. Hoda}

Editor-in-Chief

International Journal of Information Technology (BJIT) 